\title{
Successful ageing, depression and resilience research; a call for a priori approaches to investigations of resilience
}

\author{
M. Huisman ${ }^{1,2 *}$, S. S. Klokgieters ${ }^{2}$ and A. T. F. Beekman ${ }^{3}$ \\ ${ }^{1}$ Department of Epidemiology \& Biostatistics, Amsterdam Public Health Institute, VU University Medical Center, Amsterdam, The Netherlands \\ ${ }^{2}$ Department of Sociology, VU University, Amsterdam, The Netherlands \\ ${ }^{3}$ Department of Psychiatry, Amsterdam Public Health Institute, VU University Medical Center, Amsterdam, The Netherlands
}

\begin{abstract}
Given the multitude of risk factors for depression in modern society and given the negative consequences of depressive problems for successful ageing, investigating resilience in relation to depression may help identifying entry points for reducing the burden of morbidity. Research on resilience begins with the realisation that individuals may demonstrate good physical or psychological functioning despite being exposed to risk experiences that can have serious negative impact on functioning. Interest in investigating resilience within ageing research has been increasing. Among the approaches toward investigating resilience are so-called a priori approaches, where criteria for inferring resilience are established a priori. In this editorial, we highlight some of the advantages of taking a priori approaches to the study of resilience and we touch on the implications for a priori approaches for the topic of resilience and depression. We argue that depression should take a prominent role in resilience research, because depression is strongly associated with opportunities for successful ageing.
\end{abstract}

First published online 10 July 2017

Key words: Depression, elderly, epidemiology, models/theories of psychiatry.

Successful ageing and resilience - these are concepts that occupy the minds of scholars, as societies are confronted with unprecedented population ageing. The application of these concepts in empirical research generates much interest and discussion. The implications of these concepts for depression research should be debated, because good mental health is instrumental to both of them.

Successful ageing is among the core concepts of gerontology, but the operational definition of successful ageing has been heavily debated (Cosco et al. 2013). Articulated suggestions for modification of approaches to define successful ageing pointed toward the necessity to incorporate information on mental health. Most older adults value emotional functioning highly, in addition to physical functioning (Bowling \& Dieppe, 2005). Following those suggestions, studies have incorporated indicators of mental health, such as depressive symptomatology, in operational definitions of successful ageing (Kok et al. 2017). Even if depression is not considered to be part of successful ageing, it is strongly associated with it (Jeste et al. 2013).

From the perspective of public health, it has been clear that the burden of depressive problems has

* Address for correspondence: M. Huisman, Department of Epidemiology \& Biostatistics, P.O. Box 7057, 1007MB, Amsterdam, The Netherlands. (Email: ma.huisman@vumc.nl) been a considerable challenge to healthy population ageing (Murray \& Lopez, 1997). Given the multitude of risk factors for depression in modern society and given the consequences of depressive problems, investigating resilience in relation to depression seems an obvious route toward identifying entry points for reducing the burden of morbidity.

Research on resilience is the science that explains why people thrive despite being exposed to specific risk experiences. In the remainder of this editorial we refer to resilience among human beings. However, research from other academic disciplines study resilience of other systems, such as social systems, economic systems or ecological systems. Origins of resilience research can be found in different academic disciplines, such as ecology (Primm, 1984; Holling, 1986; Adger, 2000) and developmental psychology (Rutter, 1987; Garmezy, 1993; Werner, 1995; Rutter, 2006). Emblemic is the study of children growing up during the times of the Great Depression. Considered to be among key contributions to sociology and developmental psychology are the findings of Glen H. Elder, showing that an unavoidable life outcome of cumulative disadvantage following from Depression hardship was not supported by empirical evidence (Elder, 2011). Elder's work demonstrated that individuals from the Depression cohort often fared well in their adult lives, when they had access to advanced education, marriage or military training, providing opportunities 
for schooling to repair educational and skill deficiencies. These opportunities led to turning points in their lives, allowing them to overcome the potentially negative consequences of the Depression years (Elder, 1974).

Research on resilience begins with the realisation that some individuals demonstrate good physical or psychological functioning despite being exposed to risk experiences that can have serious negative impact on functioning (Rutter, 2006). For instance, few experiences can be as traumatic as child abuse. Among other things, abused children tend to report lower levels of well-being, risk behaviour (Gilbert et al. 2009) and higher levels of clinically relevant aggression (Holmes et al. 2015). Nevertheless, there are specific groups of individuals that have suffered physical abuse but remain free from clinically relevant levels of aggression. Research suggests that such children had the advantage of specific protective factors, such as good prosocial skills (Holmes et al. 2015). The idea behind research on resilience is that subgroups of resilient individuals such as these are not merely a group of statistical outliers, but have had access to psychological, social and/ or material assets and resources that helped them to deal with such experiences in such a way that they have been able to remain free from health problems later in life. Sometimes, resilience appears to be quite common, rather than being an exception (Southwick et al. 2014).

Interest in investigating resilience within ageing research has been increasing. For example, the theme of the 2012 issue of the Annual Review of Gerontology and Geriatrics was 'Emerging perspectives on resilience in adulthood and old age' (Hayslip \& Smith, 2012). It included several chapters, discussing resilience in immune functioning, mood disorders, psychological resilience and cognitive resilience and resilience and chronic illness and disability, among others. In 2011, Windle introduced the concept to gerontology with a review and concept analysis (Windle, 2011). She proposed the following definition: 'Resilience is the process of effectively negotiating, adapting to, or managing significant sources of stress or trauma. Assets and resources within the individual, their life and environment facilitate this capacity for adaptation and 'bouncing back' in the face of adversity. Across the life course, the experience of resilience will vary' (Windle, 2011).

It has been argued that the concept of resilience is so ambiguous that many may have an intuitive grasp of what it means, but that meaningful and comprehensive measurement falls short (Panter-Brick \& Leckman, 2013). A review of operational definitions of resilience in longitudinal studies indeed demonstrates that several have been in use (Cosco et al. 2017), which may lead to conceptual confusion. Different operational definitions of resilience include resilience as measured with resilience metrics, data driven latent variable models and $a$ priori established criteria of resilience. At first glance, all three approaches may have their strengths and limitations (Cosco et al. 2017). But we believe that an $a$ priori approach to establishing criteria for inferring the presence of resilience has at least two important merits, which have implications for incorporating resilience in the study of depression. We aim to highlight these merits in this editorial.

Perhaps the most important merit of an a priori approach is that the study of resilience can be geared toward the stressor, or risk exposure, that is at hand. When resilience is seen as identification of those who have better outcomes than could be expected given the circumstances (Windle, 2011), it follows that an $a$ priori approach to defining what resilience is should identify: (1) what the 'circumstances' at hand are; i.e. what is the adversity, stressor or risk exposure in which researchers are interested, and (2) when an outcome is better than could be expected. Thus, resilience equates with the presence of an a priori-selected adversity, stressor or risk factor and the presence of an $a$ priori-selected outcome that is better than expected, given exposure to the adversity.

A priori approaches should carefully define both criteria and ensure that the yardstick chosen for measuring an outcome that is better than could be expected is indeed relevant to the adversity. For example, we could be interested in investigating resilience in older people who have had a lifetime of exposure to low socioeconomic position. Such exposure is chronic, not acute. Older people can be confronted with decline in multiple domains of functioning. Not just the physical, but also the cognitive, emotional and social domains of functioning. Besides, socioeconomic adversity has been linked to adverse outcomes spanning these multiple domains. These facts are crucial for the study of resilience in the context of this particular example, because they imply that resilient older people with low socioeconomic position cannot be identified on the basis of their functioning on just one domain. A proper investigation of resilience in this example should avoid the pitfall of labelling people as resilient based on information on one domain (e.g. absence of depression), while they may be in fact performing rather poorly on other domains. A suggestion for a relevant outcome in this context could be a measure of successful ageing that encompasses information about functioning across multiple domains, including depression.

A downside of using resilience metrics, such as measurement instruments of psychological resilience, is that they consider only a limited part of all potential protective factors that may protect against a stressor in different situations. In well-known resilience metrics, 
such as the dispositional resilience scale (Bartone et al. 1989) and ego-resiliency (Block \& Kremen, 1996), resilience is viewed as a personality trait. This personality trait is considered static, independent of the disadvantage and transferrable into different environments. The trait is labelled resilient when it successfully moderates the effects of stress and promotes adaptation. While some of the more recent metrics also incorporate interpersonal elements (Connor \& Davidson, 2003; Smith et al. 2008), their predominant focus remains on psychological and individual aspects of resilience. The metrics often include aspects of personal competence, interpersonal control and self-esteem. In Windle et al. (2011) performed a review study on 15 resilience metrics published in peer-reviewed journals and criticised the lack of attention that was paid to family and community resources. They questioned whether the metrics cater sufficiently to a multidimensional definition of resilience; a definition that includes assets and resources available in the environment in addition to personality of the individual.

Therefore, a second merit of an a priori approach to research on resilience is that it allows for careful a priori consideration of the factors that may help individuals overcome the specific adversity at hand and remain free from depressive problems, or to overcome depressive problems and remain free from further negative outcomes that may be the consequence of the depressive problems. With regards to the latter, both low neuroticism and absence of pain may improve recovery from substantial depressive symptomatology in older adults (Jeuring et al. 2016), indicating that resilience factors can be both aspects of personality as well as aspects of physical functioning. Further negative consequences of depression in late life are manifold, and include comorbidity, disability, complications in comorbidity and mortality (Blazer, 2003). Likewise, adversities that may give rise to depression in late life are manifold, including biological, psychological and social risks (Blazer \& Hybels, 2005). Considering the many causes and consequences of depression in late life, it seems unlikely that there are single specific resilience factors that protect against most of these causes or protect against most negative outcomes of depression. In other words: if understanding the causes and consequences of depression requires a biopsychosocial approach, as many believe, understanding resilience in the face of depression or resilience to depression also requires a biopsychosocial approach.

Thus, a proper understanding of resilience begins with the proper conceptualisation of the adversity that is believed to confer risk and the proper conceptualisation of the outcome that defines who is resilient and who is not. Both a priori methods of describing the adversity and describing the outcome may be challenging (Panter-Brick \& Leckman, 2013). In 2005, Bowling and Dieppe asked what successful ageing is, and who should define it (Bowling \& Diepe, 2005). Perhaps we should ask this question about resilience as well, in relation to each particular adversity: what is resilience, and who should define it? Exploring what should characterise resilience in caregivers of people with dementia, Joling et al. (2015) found that research professionals had completely different views about this than caregivers themselves did. While professionals highlighted that resilience would be characterised by a high level of quality of life of the caregivers, the caregivers themselves indicated that resilience would require that the caregiver and the person with dementia maintained a good relationship (Joling et al. 2015). Also, they held partly different views about what would characterise extreme adversity in this situation, where professionals indicated that if the caregiver cared for a person with dementia with severe behavioural problems it would be the most extreme adversity. The caregivers indicated that caregivers receiving little social support and having no social activities would characterise the most extreme adversity. Clearly then, who defines what resilience is in each situation influences decisions about the yardstick that is used to identify it and the data that are needed to measure it. Face validity of resilience research substantially increases if it takes into account relevant stakeholder views about the outcomes that are valuable to pursue and promote and a proper a priori approach should consider those views, whenever possible.

Coming back to depression; it is not difficult to see that depression may be relevant for a priori approaches to study resilience in several different ways. Firstly, depression can be seen as a source of stress. Suffering from depression increases people's risk of further adverse outcomes, but there is variability in those outcomes and significant numbers of people suffering from depression may remain free from related adverse outcomes. Studying the characteristics of this group would be worthwhile from a preventative point of view. Secondly, depression is an obvious (undesirable) outcome - a yardstick that researchers may use to indicate presence of resilience (i.e. absence of depression despite exposure to a specific stressor), or resilience failure (i.e. development of depression after exposure to a stressor). Thirdly, depression may also act as a factor that might undermine resilience in the face of other types of adversity that are related to ageing, by reducing physical integrity or depleting mental and social resources.

While a priori approaches highlight the need to define the adversity as well as the outcome prior to investigation, we suggest that proper study of the process of 
resilience may not only rely on information about the outcome after the adversity, but also on information about functioning before the exposure to adversity occurred. Taking the prior situation into account may lead to identification of qualitatively distinct resilience groups. This principle is clearly demonstrated by (Galatzer-Levy \& Bonanno 2012). Studying trajectories of depression before and after bereavement, GalatzerLevy \& Bonanno identified a large group of resilient older adults who remained free from substantial depressive symptomatology, consisting of $66 \%$ of their sample. They also identified a smaller group $(10 \%)$ with substantial pre-bereavement depressive problems, but showing recovery after bereavement. In this study, bereavement was the adversity and trajectories of depression were the outcome, where trajectories reflected the process of adaptation and coping spanning periods before and after bereavement.

Finally, it remains important to realise that exposure to adversity is rarely random. The most vulnerable and least endowed people are often most likely to be exposed to adversity. In addition, they are also the ones who have the least resources or assets to successfully cope with adversity. Research on resilience and depression should prioritise finding protective factors that may increase the resilience of vulnerable groups specifically. These groups may include older populations.

\section{Acknowledgements}

Martijn Huisman is supported by a VIDI grant from the Netherlands Organisation for Scientific Research (grant number 452-11-017).

\section{References}

Adger WN (2000). Social and ecological resilience: are they related? Progress in Human Geography 24, 347-364.

Bartone PT, Ursano RJ, Wright KM, Ingrahem LK (1989). The impact of a military air disaster on the health of assistance workers: a prospective study. Journal of Nervous and Mental Disease 177, 317-328.

Blazer D (2003). Depression in late life: review and commentary. Journal of Gerontology Medical Sciences 58A, 249-265.

Blazer DG, Hybels CF (2005). Origins of depression in later life. Psychological Medicine 35, 1241-1252.

Block J, Kremen AM (1996). IQ and ego-resiliency: conceptual and empirical connections and separateness. Journal of Personality and Social Psychology 70, 349-361.

Bowling A, Dieppe P (2005). What is successful ageing and who should define it? British Medical Journal 331, 1548-1551.

Connor KM, Davidson JR (2003). Development of a new resilience scale: the Connor-Davidson resilience scale (CD-RISC). Depression and Anxiety 18, 76-82.

Cosco TD, Prina AM, Perales J, Stephan BCM, Brayne C (2013). Operational definitions of successful aging: a systematic review. International Psychogeriatrics 26, 1-9.
Cosco TD, Kaushal A, Hardy R, Richards M, Kuh D, Stafford M (2017). Operationalising resilience in longitudinal studies: a systematic review of methodological approaches. Journal of Epidemiology and Community Health 71, 98-104.

Elder GH (1974). Children of the Great Depression: Social Change in Life Experience. Chicago: University of Chicago Press.

Elder GH (2011). Families, social change and individual lives. Marriage and Family Review 31, 187-203.

Galatzer-Levy IR, Bonnanno GA (2012). Beyond normality in the study of bereavement: heterogeneity in depression outcomes following loss in older adults. Social Science $\mathcal{E}$ Medicine 74, 1987-1994.

Garmezy N (1993). Children in poverty; resilience despite risk. Psychiatry 56, 127-136.

Gilbert R, Widom CS, Browne K, Fergusson D, Webb E, Janson $S$ (2009). Burden and consequences of child maltreatment in high-income countries. Lancet 373, 68-81.

Hayslip B, Smith GC (ed.) (2012). Emerging perspectives on resilience in adulthood and later life. Annual Review of Gerontology and Geriatrics 32.

Holling CS (1986). The resilience of terrestrial ecosystems: local surprise and global change. Sustainable Development of the Biosphere 14, 292-317.

Holmes MR, Yoon S, Voith LA, Kobulsky JM, Steigerwald S (2015). Resilience in physically abused children: protective factors for aggression. Behavioral Science 5, 176-189.

Jeuring HW, Huisman M, Comijs HC, Stek ML, Beekman ATF (2016). The long-term outcome of subthreshold depression in later life. Psychological Medicine 46, 2855-2865.

Jeste DV, Savla GN, Thompson WK, Vahia IV, Glorioso DK, Martin AS, Palmer BW, Rock D, Golshan S, Kraemer HC, \& Depp CA (2013). Older age is associated with more successful aging: role of resilience and depression. American Journal of Psychiatry 170, 188-196.

Joling KJ, Windle G, Droes R, Huisman M, Hertogh CMPM, Woods RT (2015). What are essential features of resilience for informal caregivers of people living with dementia? A Delphi consensus examination. Aging and Mental Health, 1-9.

Kok AAL, Aartsen MJ, Deeg DJH, Huisman M (2017). Capturing the diversity of successful aging: an operational definition based on 16-year trajectories of functioning. Gerontologist 57, 240-251.

Murray CJ, Lopez AD (1997). Alternative projections of mortality and disability by cause 1990-2020: Global Burden of Disease Study. Lancet 349, 1498-1504.

Panter-Brick C, Leckman JF (2013). Editorial commentary: resilience in child development - interconnected pathways to wellbeing. Journal of Child Psychology and Psychiatry 54, 333-336.

Primm SL (1984). The complexity and stability of ecosystems. Nature 307, 321-326.

Rutter M (1987). Psychosocial resilience and protective mechanisms. American Journal of Orthopsychiatry 57, 316-331.

Rutter M (2006). Implications of resilience concepts for scientific understanding. Annals of the New York Academy Sciences 1094, 1-12.

Smith BW, Dalen J, Wiggins K, Tooley E, Christopher P, Bernard J (2008). The brief resilience scale: assessing the 
ability to bounce back. International Journal of Behavioral Medicine 15, 194-200.

Southwick SM, Bonanno GA, Masten SA, Panter-Brick C, Yehuda R (2014). Resilience definitions, theory, and challenges: interdisciplinary perspectives. European Journal of Psychotraumatology 5. doi: 10.3402/ejpt. v5.25338.
Werner EE (1995). Resilience in development. Current Directions in Psychological Science 4, 81-85.

Windle G (2011). What is resilience? A review and concept analysis. Review of Clinical Gerontology 21, 152-169.

Windle G, Bennett KM, Noyes JA (2011). Methodological review of resilience measurement scales. Health and Quality of Life Outcomes 9, 1-18. 